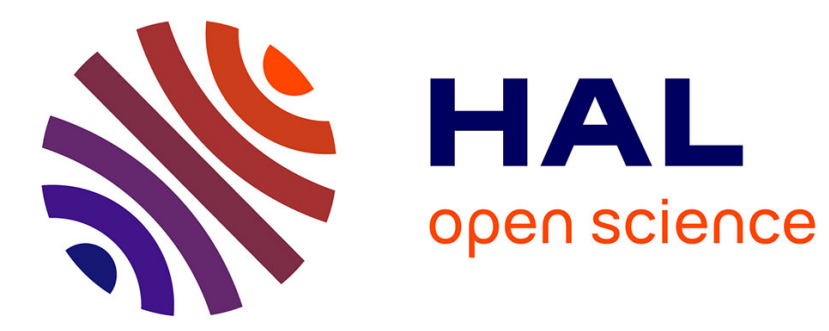

\title{
Channel characterization for optical extra-WBAN links considering local and global user mobility
}

Oussama Haddad, Mohammed Ali Khalighi, Stanislav Zvanovec

\section{To cite this version:}

Oussama Haddad, Mohammed Ali Khalighi, Stanislav Zvanovec. Channel characterization for optical extra-WBAN links considering local and global user mobility. SPIE OPTO, Feb 2020, San Francisco, United States. 10.1117/12.2544901 . hal-02475772

\section{HAL Id: hal-02475772 \\ https://hal.science/hal-02475772}

Submitted on 27 Mar 2020

HAL is a multi-disciplinary open access archive for the deposit and dissemination of scientific research documents, whether they are published or not. The documents may come from teaching and research institutions in France or abroad, or from public or private research centers.
L'archive ouverte pluridisciplinaire HAL, est destinée au dépôt et à la diffusion de documents scientifiques de niveau recherche, publiés ou non, émanant des établissements d'enseignement et de recherche français ou étrangers, des laboratoires publics ou privés. 


\title{
Channel Characterization for Optical Extra-WBAN Links Considering Local and Global User Mobility
}

\author{
Oussama Haddad $^{\mathrm{a}}$, Mohammad-Ali Khalighi ${ }^{\mathrm{b}}$, and Stanislav Zvanovec ${ }^{\mathrm{c}}$ \\ a,b Aix-Marseille University, CNRS, Centrale Marseille, Institut Fresnel, Marseille, France \\ ${ }^{\mathrm{c} D e p a r t m e n t ~ o f ~ E l e c t r o m a g n e t i c ~ F i e l d, ~ F a c u l t y ~ o f ~ E l e c t r i c a l ~ E n g i n e e r i n g, ~ C z e c h ~ T e c h n i c a l ~}$ \\ University in Prague, Prague, Czech Republic
}

\begin{abstract}
We investigate channel characterization for wireless body-area networks (WBANs) in medical applications using optical signal transmission. More specifically, we focus on uplink communication from a central coordinator node $(\mathrm{CN})$, placed on the patient's body, to an access point (AP) in a typical hospital room, which is usually referred to as extra-WBAN link. Using a ray-tracing based approach, we quantify the main characteristics of the optical channel while considering the effects of body shadowing and mobility (accounting for body movements and user global mobility inside the room). Based on the presented results, we discuss the impact of the positions of the $\mathrm{CN}$ and the AP on the link parameters. We also evaluate the link performance based on the outage probability criterion, and further quantify the performance improvement achieved by using multiple APs in the room.
\end{abstract}

Keywords: Wireless body-area networks; optical wireless communications; optical channel characterization; user mobility.

\section{INTRODUCTION}

E-Health solutions are gaining increasing popularity in a number of application scenarios as efficient means for improving the quality of life and reducing health-care costs. ${ }^{1}$ Typical examples include real-time monitoring of patients in health-care centers and tele-monitoring post-operative patients or elderly people at home. These can be realized through the use of medical wireless body-area networks (WBANs) for sending timely data from several medical sensors. These sensors are typically connected to a coordinator node $(\mathrm{CN})$, placed on the patient's body. The collected data at the $\mathrm{CN}$ is then sent to a mobile device or to a fixed access-point (AP) through what is called an extra-WBAN link.

Whereas a number of solutions have been proposed so far based on the radio-frequency (RF) technology mostly in the unlicensed frequency bands, the considerations of electromagnetic interference, data security, and the possible impact of RF waves on the tissues makes optical wireless communications of particular interest within this context. ${ }^{2,3}$

We focus in this paper on extra-WBAN uplink communication, i.e., from the $\mathrm{CN}$ to the AP, and consider the characterization of the wireless optical channel by estimating the channel impulse response (CIR). An important point here is to take into account user mobility, which can considerably affect the link availability and performance. In this sense, most previous works have been based on simplified models in order to reduce the computational complexity. For instance, ${ }^{4,5}$ considered channel characterization for optical extra-WBAN links using a Monte-Carlo ray-tracing based simulation tool called Rapsor. In, ${ }^{4}$ downlink extra-WBAN transmission was studied while neglecting local body movements and assuming that the $\mathrm{Rx}(\mathrm{CN})$ is at a distance of $29 \mathrm{~cm}$ from the body. Also, the effect of patient's body was neglected in. ${ }^{5} \mathrm{In},{ }^{6,7}$ user mobility was taken into account based on random user positions and random orientations of the sources and the detectors, considering uniform distributions. In contrast to these works, which consider simplified models, our study is based on more accurate and more realistic models with an acceptable level of details, in particular, concerning the body 3D shape and user mobility. More specifically, we propose a dynamic model based on 3D animation of a walk cycle to model body movement and random trajectories based on a modified random waypoint (RWP) mobility model. From

E-mails: Oussama.Haddad@fresnel.fr, Ali.Khalighi@fresnel.fr, xzvanove@fel.cvut.cz 


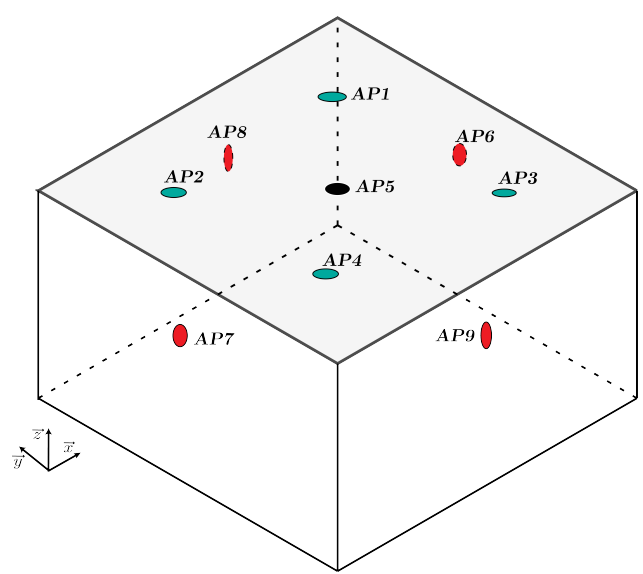

(a)

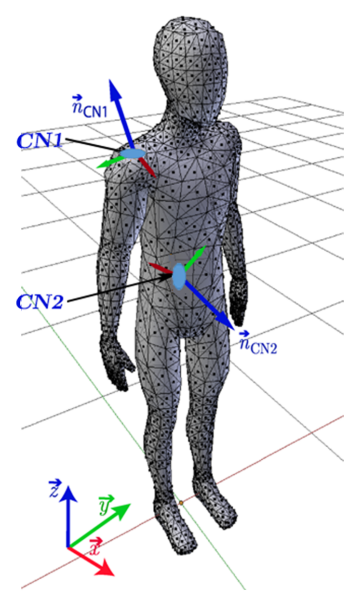

(b)

Figure 1. Illustration of the considered placements for (a) the APs and (b) the CNs.

the simulated CIRs, we study different link metrics including channel DC gain and delay spread. We also present a stability analysis of the channel using the auto-correlation function (ACF) and the channel coherence time criteria. Moreover, considering the simple on-off keying (OOK) modulation, we evaluate the link performance in terms of outage probability and investigate the benefit of using multiple APs in the room.

The remainder of the paper is organized as follows. Section 2 presents the main assumptions on the considered WBAN link and the methodology for simulations. Numerical results are then presented in Section 3 to study different optical link metrics. Afterwards, we evaluate the outage probability of the link in Section 4 and investigate the performance gain offered by using multiple APs. Section 5 concludes the paper.

\section{ASSUMPTIONS AND SIMULATION APPROACH}

\subsection{General Assumptions}

Consider an extra-WBAN link between a CN on the patient's body and an AP. We consider two different placements for the $\mathrm{CN}$, i.e., on the shoulder (the Rx pointing upward) and on the waist (the Rx pointing horizontally ahead), as suggested in, ${ }^{8}$ see Fig.1(b). For the former case, the communication link would be less affected by body shadowing when the AP is placed on the ceiling. We consider an LED at the transmitter (Tx) side, i.e., at the CN, emitting on infrared wavelength of $\lambda_{0}=850 \mathrm{~nm}$ with a bandwidth of $\Delta \lambda=30 \mathrm{~nm}$. Without loss of generality, we consider a Lambertian pattern ${ }^{3}$ for the LED. Also, in order to ensure minimum beam blockage, we consider a Lambertian order of $m=1$, corresponding to a semi-angle of $60^{\circ}$. For the sake of simplicity, we assume an empty room, i.e., without any furniture or equipment.

At the receiver $(\mathrm{Rx})$ side, i.e., at the $\mathrm{AP}$, we consider a simple PIN photo-detector of $1 \mathrm{~cm}^{2}$ active area without any lens and with a large field-of-view (FOV) of $60^{\circ}$ (semi-angle). Note that the assumption of such a relatively large active area is justified by the fact that relatively low data-rates, on the order of $1 \mathrm{Mbps}$ at most, are required in medical WBANs. ${ }^{9}$ To investigate the practical interest of using more than one AP at the $\mathrm{Rx}$, we also consider the case where several APs are used in order to minimize beam blockage probability. For this, multiple APs will be considered either on the ceiling or on the walls of the room. Then, the received signals are combined based on equal-gain combining (EGC) or selection combining (SC, i.e., taking the strongest signal) strategies. ${ }^{10}$ Figure 1 shows the top view of the room with the considered positions for the APs, and also illustrates the considered positions of the CN on the body. As it can be seen, AP1-AP5 are symmetrically placed on the ceiling and point downward. Also, AP6-AP9 are placed at half width and $2 \mathrm{~m}$ height and point straight toward the center of the room. Note that, by default, we consider the case of AP5 (i.e., a single AP at the center of the ceiling). 
Table 1. Simulation parameters.

\begin{tabular}{|c|c|c|}
\hline \multirow{3}{*}{ Tx (CN) } & $m$ & 1 \\
\cline { 2 - 3 } & $\lambda_{0}$ & $850 \mathrm{~nm}$ \\
\cline { 2 - 3 } & $\Delta \lambda$ & $30 \mathrm{~nm}$ \\
\hline \hline \multirow{3}{*}{$\operatorname{Rx}(\mathrm{AP})$} & FOV & $60^{\circ}$ \\
\cline { 2 - 3 } & Active Area & $1 \mathrm{~cm}^{2}$ \\
\cline { 2 - 3 } & $\mathcal{R}$ & $0.65 \mathrm{~A} / \mathrm{W}$ \\
\hline \hline \multirow{3}{*}{$\operatorname{Room}^{12}$} & Dimensions & $(5 \times 5 \times 3) \mathrm{m}^{3}$ \\
\cline { 2 - 3 } & Walls Reflectivity & $0.83($ Plaster $)$ \\
\cline { 2 - 3 } & Floor Reflectivity & $0.87($ Pinewood $)$ \\
\cline { 2 - 3 } & Ceiling Reflectivity & $0.83($ Plaster $)$ \\
\hline \hline \multirow{2}{*}{ Body } & Dimensions & $(1.7 \times 0.3 \times 0.2) \mathrm{m}^{3}$ \\
\cline { 2 - 3 } & Reflectivity & $0($ absorbing $)$ \\
\hline \hline
\end{tabular}

Denoting the CIR by $h(t)$, we will in particular study the channel DC gain $H_{0}$ and the root mean squared (RMS) delay spread $\tau$, defined in the following. ${ }^{3}$

$$
H_{0}=\int h(t) \mathrm{d} t, \quad \tau=\sqrt{\frac{\int_{0}^{\infty}\left(t-\varepsilon_{0}\right)^{2} h^{2}(t) \mathrm{d} t}{\int_{0}^{\infty} h^{2}(t) \mathrm{d} t}} .
$$

Here, $\varepsilon_{0}$ denotes the mean excess delay spread, given by: ${ }^{3}$

$$
\varepsilon_{0}=\frac{\int_{0}^{\infty} t h^{2}(t) \mathrm{d} t}{\int_{0}^{\infty} h^{2}(t) \mathrm{d} t} .
$$

To characterize channel time variations, we consider the channel coherence time. This is approximated by the point where the ACF falls below a certain value, e.g., 0.5. We will denote this by $T_{c}$.

Moreover, in order to quantify the link performance, we consider the outage probability $P_{\text {out }}$, which is defined as the probability that the bit-error-rate (BER) for a given channel realization is higher than a pre-defined threshold $\mathrm{BER}_{\mathrm{th}}$. We assume negligible background noise effect at the Rx, which can be justified assuming the use of a narrow-band optical filter.

\subsection{Simulations Methodology}

To estimate the CIR, we use ray-tracing based Monte-Carlo simulations using Opticstudio software from Zemax. ${ }^{11}$ We set the reflection properties of the different surfaces according to ${ }^{12}$ and consider at least 3 order reflections for beam propagation. To design the 3D scene for the simulator, we use the Blender software. We then use a Python script to load a set of configurations describing the user movements and orientations for a set of consecutive snapshots of a walk scenario. Afterwards, the CIR for different user positions and body configurations are calculated using Opticstudio, which we then use to analyze different channel metrics.

To study the effect of user mobility, we take into account both the change in the position of the user inside the room and the movement of the body parts, i.e., the arms, legs, etc. Concerning the former, which we will refer to as global user mobility, we have simulated a walk trajectory based on a modified RWP model. In contrast to the RWP model according to which the user moves in a straight line from one point to another, ${ }^{13}$ here we consider the movements of the user along a curved path by interpolating the waypoints using a 3-order polynomial. This provides a more realistic trajectory by avoiding sharp rotations. To consider the movements of the body parts, that we will call local mobility, we use a sampled walk animation sequence. Table 1 summarizes the parameters we have used in our simulations. 

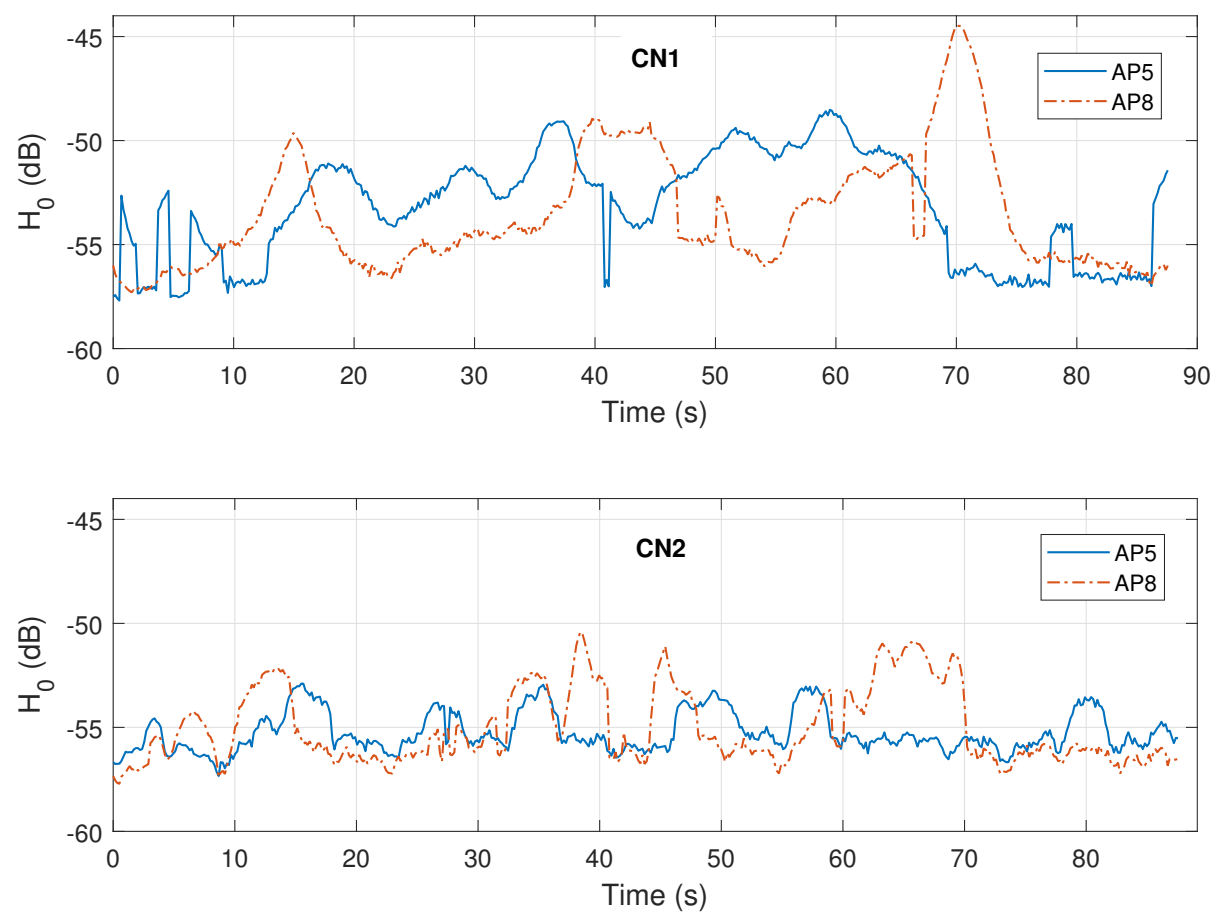

Figure 2. $H_{0}$ variations for the cases of $\mathrm{CN} 1$ and $\mathrm{CN} 2$ in the single AP configuration for AP5 and AP8 positions (see Fig.1).

\section{CHANNEL CHARACTERIZATION, NUMERICAL RESULTS}

\subsection{Channel DC gain}

One of the most important channel metrics is its DC gain that directly determines the signal-to-noise ratio (SNR) at the $\mathrm{Rx}$.

\subsubsection{Single AP Configurations}

Consider first the case of a single AP. Figure 2 shows the evolution of $H_{0}$ (in dB) for AP5 and AP8 as the Rx, as well as for the two cases of CN1 and CN2 (see Fig. 1). The abscissa represents the simulation time, assuming a walk speed of $0.45 \mathrm{~m} / \mathrm{s}$.

We observe from these results that, reasonably, we have a larger $H_{0}$ on average for the case of CN1 (located on the shoulder), compared to CN2 (located on the waist). Interestingly, the average $H_{0}$ is more or less the same, regardless of the position of the AP: the mean $H_{0}$ corresponding to $\mathrm{CN} 1$ and $\mathrm{CN} 2$ cases is around -53 and $-55 \mathrm{~dB}$, respectively (for both AP5 and AP8). An important result is that we notice larger $H_{0}$ variations and longer mean time between fades (MTBFs) for CN1, compared to CN2. For instance, with AP5, the range of variations of $H_{0}$ are around 9.2 and $4.4 \mathrm{~dB}$, for $\mathrm{CN} 1$ and $\mathrm{CN} 2$ cases, respectively. The corresponding standard deviation is about $3 \mathrm{~dB}$ for $\mathrm{CN} 1$, and it ranges between 1 to $2 \mathrm{~dB}$ for $\mathrm{CN} 2$.

Larger $H_{0}$ variations are observed for $\mathrm{AP} 8$, with the range of variations of $H_{0}$ about 12.8 and $7.3 \mathrm{~dB}$, for $\mathrm{CN} 1$ and CN2 cases, respectively. These relatively larger variations can be explained by the fact that AP8 (placed on the wall) is more sensitive to body shadowing resulting from body rotations during the walk trajectory (compared with AP5).

\subsubsection{Multiple AP Configurations}

Consider now the case where four APs are used for signal reception. Two configurations are studied: where the APs are placed on the ceiling (i.e., AP1 to AP4) or on the walls (i.e., AP6 to AP9), see Fig. 1. Based on EGC 

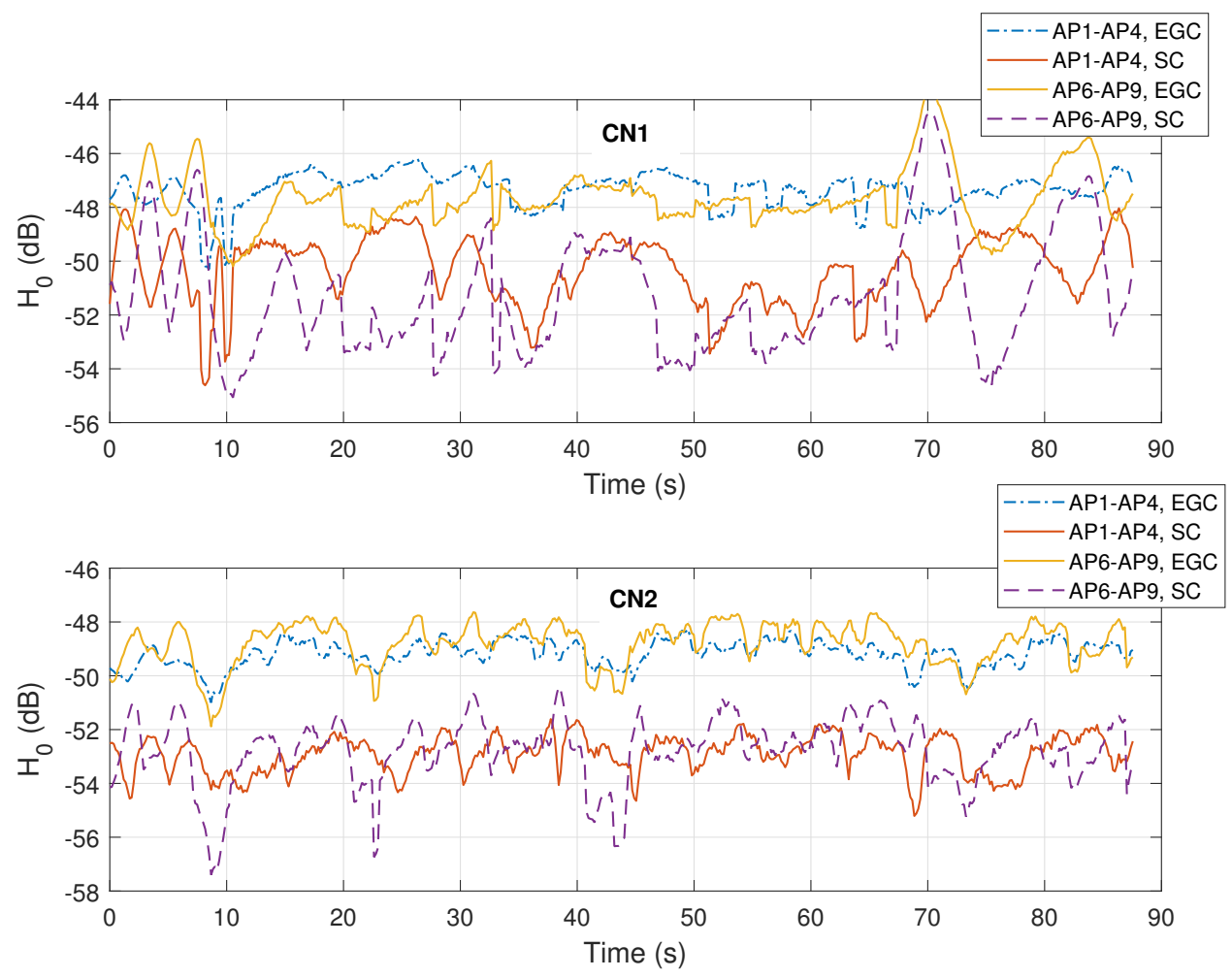

Figure 3. $H_{0}$ variations for the cases of $\mathrm{CN} 1$ and $\mathrm{CN} 2$ in the multiple-AP configurations using EGC and SC detection methods.

or SC combination methods, we have shown the variations of the channel DC gain in Fig. 3 for the cases of CN1 and CN2. As expected, using multiple APs allows an increase in the mean $H_{0}$ and a reduction of its standard deviation, compared to the single AP configuration. Obviously, compared with SC, the EGC method (which provides performance close to the optimal maximal-ratio combining ${ }^{10}$ ) provides an improved performance, i.e., a higher average and a lower standard deviation for $H_{0}$.

Compared with single AP configurations, here we have an improvement of the mean $H_{0}$ of 5.8 to $6.2 \mathrm{~dB}$ with EGC, and around 2.2 to $2.8 \mathrm{~dB}$ with SC (for both cases of AP1-AP4 and AP6-AP9). Meanwhile, similar to the case of single AP, we notice a higher average channel gain (about 1.1 to $2.6 \mathrm{~dB}$ ) and larger variations (about 1.4 to $3.6 \mathrm{~dB}$ ) for the case of CN1. Also, larger variations and shorter MTBFs are observed for the APs on the walls, compared with the APs on the ceiling.

\subsection{Channel RMS Delay Spread}

Channel delay spread is the key factor for quantifying channel frequency selectivity and the need to channel equalization at the Rx. ${ }^{14}$ From the simulated CIRs, we have calculated the mean and the standard deviation of the RMS delay spread, denoted by $\bar{\tau}$ and $\sigma_{\tau}$, respectively. Results are presented in Table 2 for single and multiple AP configurations and the cases of $\mathrm{CN} 1$ and $\mathrm{CN} 2$.

For the single AP configurations, $\bar{\tau}$ ranges between 5.2 to $10.2 \mathrm{~ns}$, with $\sigma_{\tau}$ between 1.9 and $4.4 \mathrm{~ns}$. For AP5, we notice a smaller $\bar{\tau}$ with $\mathrm{CN} 1$, whereas a smaller $\bar{\tau}$ is obtained for AP8 with CN2. Comparing the cases of CN1 and $\mathrm{CN} 2$, we generally notice shorter $\bar{\tau}$ for the former, and lower $\sigma_{\tau}$ for the latter. A shorter $\bar{\tau}$ can be explained by a less shadowing impact on the direct (LOS, line-of-sight) path, given the random orientations and positions of the Tx.

For the multiple AP configurations, we notice the same trend and the same range of $\bar{\tau}$ for the cases of CN1 and $\mathrm{CN} 2$ as for the single AP configurations. Nevertheless, with EGC detection, we have a quite smaller $\sigma_{\tau}$, especially for APs on the ceiling, but it is the inverse for SC detection, which is again rational. 
Table 2. Mean and standard deviation (in ns) of the RMS delay spread.

\begin{tabular}{|c|c|c||c|c||c|c|}
\hline \multicolumn{2}{|c|}{ Configuration } & \multicolumn{2}{c||}{ CN1 } & \multicolumn{2}{c|}{ CN2 } \\
\cline { 4 - 7 } Single AP & \multicolumn{2}{|c|}{ Ceiling AP5) } & 5.2 & 4.4 & 10.2 & 1.9 \\
\cline { 2 - 7 } & \multicolumn{2}{|c|}{ Wall (AP8) } & 7.3 & 4.2 & 8.3 & 3.7 \\
\hline \hline \multirow{3}{*}{ Multiple APs } & \multirow{2}{*}{ Ceiling (AP1-AP4) } & EGC & 4.7 & 1.6 & 11.3 & 0.8 \\
\cline { 3 - 7 } & & SC & 7.9 & 3.8 & 7.1 & 3.6 \\
\cline { 2 - 7 } & \multirow{2}{*}{ Walls (AP6-AP9) } & EGC & 6.1 & 4.2 & 8.6 & 2.9 \\
\cline { 3 - 7 } & & SC & 7.4 & 3.0 & 9.5 & 1.9 \\
\hline
\end{tabular}

Table 3. Estimated channel coherence time $T_{c}$ (in sec) corresponding to the $50 \%$ of the ACF, based on the simulated user trajectory.

\begin{tabular}{|c|c|c||c|c|}
\hline \multicolumn{2}{|c|}{ Configuration } & CN1 & CN2 \\
\hline \hline \multirow{2}{*}{ Single AP } & \multicolumn{2}{|c||}{ Ceiling } & 4.2 & 1.4 \\
\cline { 2 - 5 } & \multicolumn{2}{|c|}{ Wall } & 2.3 & 2.0 \\
\hline \hline \multirow{3}{*}{ Multiple AP } & \multirow{2}{*}{ Ceiling } & EGC & 1.5 & 1.0 \\
\cline { 3 - 5 } & & SC & 1.4 & 0.9 \\
\cline { 2 - 5 } & \multirow{2}{*}{ Walls } & EGC & 1.5 & 1.0 \\
\cline { 3 - 5 } & & SC & 1.6 & 1.0 \\
\hline
\end{tabular}

Overall, from these results and assuming simple OOK modulation, we can conclude that the communication channel can be considered as effectively frequency non-selective for data-rates up to approximately $10 \mathrm{Mbps}$, regardless of the position of the Tx. When $\mathrm{AP}(\mathrm{s})$ is (are) positioned on the ceiling, this condition is verified even for higher data-rates.

\subsection{Channel Coherence Time}

Channel coherence time $T_{c}$ is a useful metric for characterizing channel time variations. It determines, for instance, the MAC layer parameters for setting the frame length and the transmission of training sequences. As explained in Section 2, to approximate $T_{c}$, we consider the delay where the ACF falls by $50 \%$. The corresponding values are presented in Table 3. We have also presented the ACF plots for the different AP configurations and the cases of CN1 and CN2 in Fig. 4. The interesting point is the substantial reduction of the coherence time for the multiple-AP configurations. In particular, a reduction of factor 3 is observed for the case of CN1 with respect to the AP5 configuration. This means that despite the benefit of spacial diversity in reducing channel fading, we have a degraded channel stability due to a higher dynamic for the ensemble of underlying channels.

Focusing on the ACF plots, we further notice a different trend for CN1 and CN2 cases, for AP5 and AP8 configurations. These results are consistent with those previously presented for $H_{0}$ in Subsection 3.1. For instance, for AP5 with CN1, the LOS link is occasionally shadowed by the head of the user during relatively long periods, thus a more spread ACF and a longer $T_{c}$. Conversely, the LOS between AP5 and CN2 is more frequently subject to shadowing by the arms, resulting in more variations of $H_{0}$, thus a more compact ACF and a shorter $T_{c}$. Similar trends are observed for the multiple AP configurations (see the low values of ACF on the plots). Here, focusing on $T_{c}$, we notice close values for for CN1 and CN2, independently of the position of the APs (i.e., on the ceiling or on the walls). Also, the SC method gives a slightly longer $T_{c}$.

\section{LINK PERFORMANCE ANALYSIS}

After presenting the main channel characterization metrics, we provide here a preliminary analysis of the link performance for the case of user mobility. Given the random time-varying nature of the channel, a relevant performance metrics is the outage probability $P_{\text {out }}$, which is defined as the probability that the instantaneous link BER falls below a pre-defined threshold BER $\mathrm{th}_{\mathrm{th}}$. In order not to resort to time consuming simulations with 


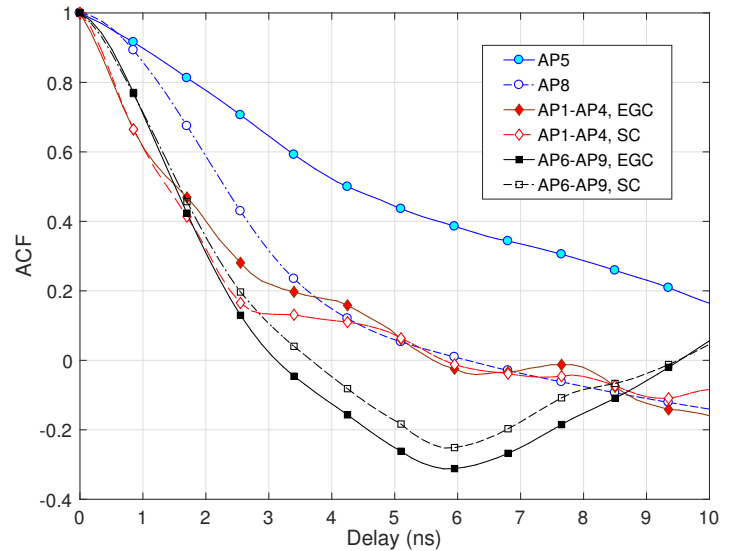

(a)

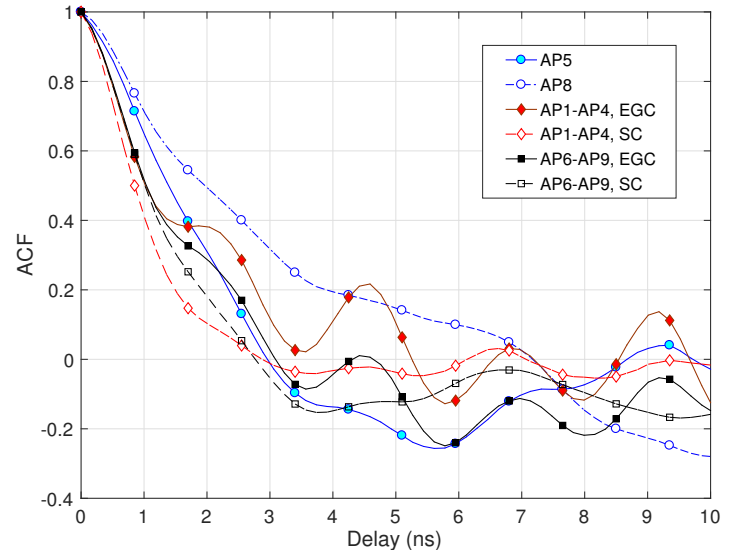

(b)

Figure 4. Calculated ACF for the cases of (a) CN1 and (b) CN2 and different AP configuration based on the simulated user trajectory.

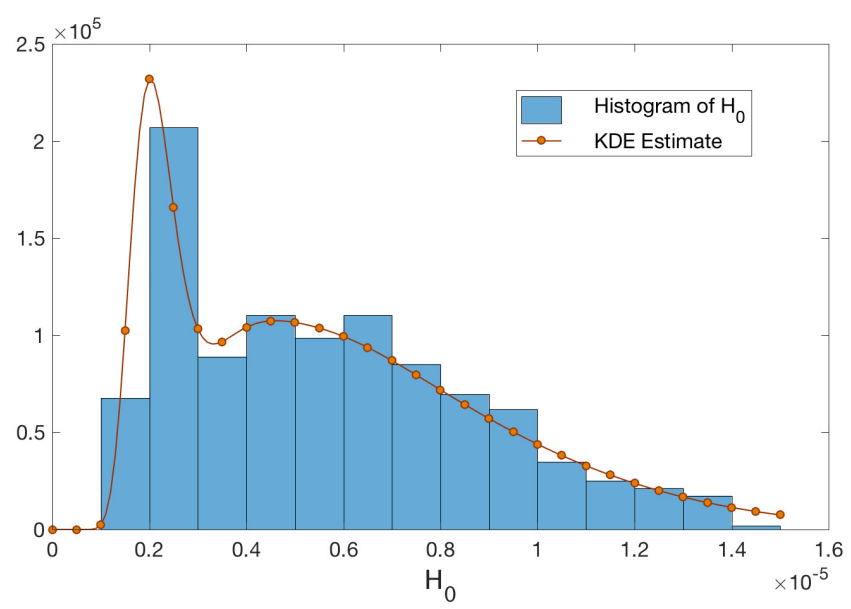

Figure 5. Histogram of $H_{0}$ for 520 channel realizations for the case of CN1 and AP5 and estimated PDF by KDE with Gaussian kernel.

Opticstudio, we propose here a statistical model for the channel DC gain. This is obtained based on the nonparametric kernel density estimation (KDE), applied to the obtained results for the simulated user trajectory (see the previous section).

Given a set of $N$ realizations $x_{i}$ of a random variable $x$ with probability density function $(\mathrm{PDF}) f_{X}(x)$, the KDE estimate of the PDF is given by: ${ }^{15}$

$$
\hat{f}_{X}(x)=\frac{1}{N \delta} \sum_{i=1}^{N} \mathcal{K}\left(\frac{x-x_{i}}{\delta}\right),
$$

where $\mathcal{K}$ is the kernel and $\delta$ is the smoothing parameter, also called bandwidth. For the sake of simplicity, we consider here the case of a Gaussian kernel. We have presented in Fig. 5 for the case of CN1 and AP5 the histogram of the simulated $H_{0}$ together with the fitted PDF based on KDE. The corresponding $\delta$ is 0.18 , which is calculated from ${ }^{16} \delta=1.06 \hat{\sigma} N^{-1 / 5}$, where $\hat{\sigma}$ is the estimated standard variation of $\log (x)$ related to (3), and $N=520$.

Using this statistical model, we have randomly generated $10^{7}$ values for $H_{0}$. Then, for each channel realization, we calculate the BER for an uncoded OOK-based link for a given data-rate $R_{b}$ and transmit power $P_{t}$. Note that we consider intensity levels of 0 and $P_{t}$ for Off and On OOK symbols, respectively. The BER is calculated 


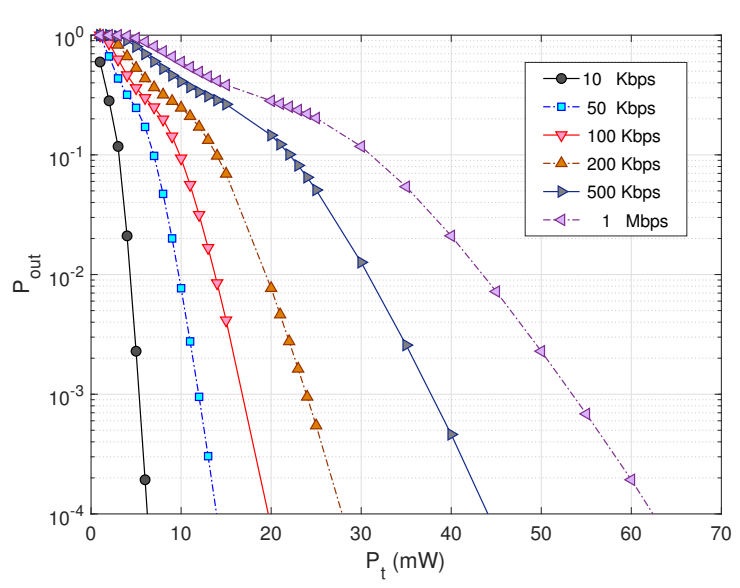

(a)

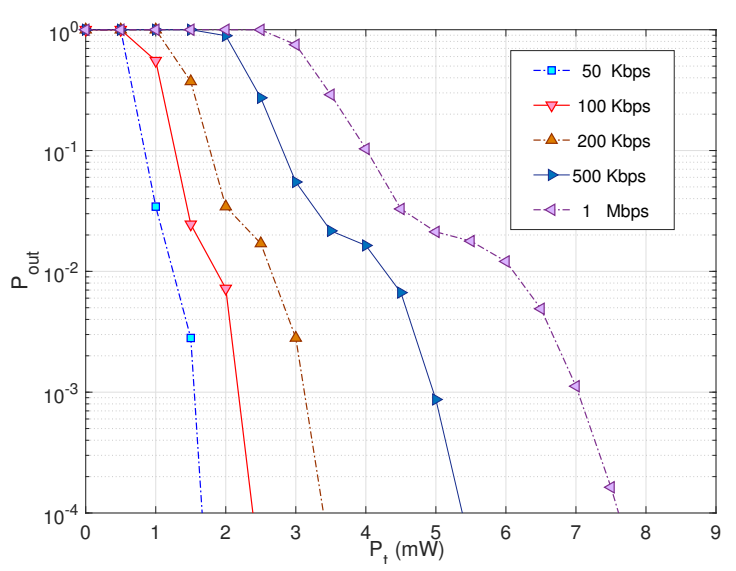

(b)

Figure 6. $P_{\text {out }}$ versus the transmit power $P_{t}$ for different data-rates $R_{b}$ for the cases of (a) CN1 and AP5, and (b) CN1 and AP1-AP4 with EGC detection. Uncoded OOK modulation.

using the following equation. ${ }^{3}$

$$
\mathrm{BER}=Q(\sqrt{\mathrm{SNR}}), \quad \text { with } \mathrm{SNR}=\frac{\left(\mathcal{R} H_{0} P_{t}\right)^{2}}{\sigma_{\mathrm{th}}^{2}},
$$

where we have assumed that the dominant noise at the $\mathrm{Rx}$ is the thermal noise with variance $\sigma_{\mathrm{th}}^{2}=4 K T_{e} B / R_{L}$ with $K$ being the Boltzman constant, $T_{e}=298 \mathrm{~K}$ the equivalent noise temperature, $B$ the system bandwidth, and $R_{L}$ the load resistance of the trans-impedance amplifier, set to $50 \Omega{ }^{17}$ Parameter $B$ is mainly determined by the $\mathrm{Rx}$ low-pass filter and is typically approximated by $R_{b} / 2$. Also, in (4), $\mathcal{R}$ stands for the photo-detector responsivity, which is set to $0.65 \mathrm{~A} / \mathrm{W}$, and $Q(x)=\frac{1}{\sqrt{2 \pi}} \int_{x}^{\infty} \exp \left(-u^{2} / 2\right) d u$.

For $P_{\text {out }}$ calculation, we consider $\mathrm{BER}_{\mathrm{th}}=10^{-3}$. We have shown in Fig. 6 plots of $P_{\text {out }}$ versus $P_{t}$ for $R_{b}$ ranging between $10 \mathrm{Kbps}$ to $1 \mathrm{Mbps}$. We have considered two cases of the link between CN1 and AP5, Fig. 6(a), and the link between CN1 and AP1 to AP4 with EGC signal detection, Fig. 6(b). Let us first consider the case of the single AP. We notice a degradation of $P_{\text {out }}$ with the increase in $R_{b}$. Indeed, with increased link data-rate, and consequently, increased $\mathrm{Rx}$ noise variance (remember that the noise variance is proportional to $R_{b}$ ), a higher BER is obtained for a given $H_{0}$. Obviously, for increased $P_{t}$, a lower $P_{\text {out }}$ is obtained.

Now let us consider the case of multiple APs. We see from Fig. 6(b) that a considerably lower transmit power is required for a target $P_{\text {out }}$, for a given data-rate. For instance, for $P_{\text {out }}=10^{-3}$ and $R_{b}=500 \mathrm{Kbps}$, the required transmit power is around 5 and $37 \mathrm{~mW}$ for the cases of four-AP and single-AP, respectively. This elucidates the substantial improvement achieved in the link reliability offered by spatial diversity. In fact, by comparing these results with Fig.6(a), we can clearly see the impact of channel gain variations due to user mobility on the link performance. Lastly, note that in Fig.6(b) for $R_{b}=10 \mathrm{Kbps}, P_{\text {out }}$ is very low even for $P_{t}=1 \mathrm{~mW}$ (that is why the corresponding plot is not seen in the figure).

\section{CONCLUSIONS}

This paper considered channel characterization for an optical-based extra-WBAN uplink for medical applications. Considering the typical case of a patient in a hospital room, we proposed an accurate approach for simulating body local and global movements and calculated the CIR. Different channel metrics were quantified, giving insight into the practical design of such systems. In particular, we studied the impact of the position of the $\mathrm{CN}$ on the patient body, that could be related to the practical implementation or user comfort reasons. Also, we showed the substantial improvement achieved in the link reliability when using multiple APs at the Rx, which is due to a significant reduction of beam shadowing during user movements. Although the use of multiple 
APs increases the implementation complexity of the network, it can be justified given the achieved performance improvement, in particular, regarding the substantial reduction of the required transmit power.

\section{Acknowledgment}

This work has been supported by VisIoN, a European project funded by the European Union's Horizon 2020 research and innovation program under the Marie Skłodowska-Curie Grant Agreement No. 764461.

\section{REFERENCES}

[1] Botsis, T. and Hartvigsen, G., "Current status and future perspectives in telecare for elderly people suffering from chronic diseases," Journal of Telemedicine and Telecare 14, 195-203 (Jan. 2008).

[2] Haddad, O. and Khalighi, M. A., "Enabling communication technologies for medical wireless body-area networks," in [Global LiFi Congress (GLC)], 1-5 (June 2019). Paris, France.

[3] Ghassemlooy, Z., Alves, L. N., Zvànovec, S., and Khalighi, M. A., eds., [Visible Light Communications: Theory and Applications], CRC-Press (2017).

[4] Behlouli, A., Combeau, P., Sahuguède, S., Julien-Vergonjanne, A., Le Bas, C., and Aveneau, L., "Impact of physical and geometrical parameters on visible light communication links," in [2017 Advances in Wireless and Optical Communications (RTUWO)], 73-76 (Nov. 2017). Riga, Latvia.

[5] Le Bas, C., Sahuguède, S., and Julien-Vergonjanne, A., "Theoretical and experimental approach for the design of an optical wireless physical activity monitoring system," International Journal of Wireless Information Networks 24, 65-77 (June 2017).

[6] Dhatchayeny, D. R., Arya, S., and Chung, Y. H., "Patient mobility support for indoor non-directed optical body area networks," Sensors 19(10) (2019).

[7] Hoang, T. B., Sahuguède, S., and Julien-Vergonjanne, A., "Optical wireless network design for off-bodysensor based monitoring," Wireless Communications and Mobile Computing (5473923), 1-13 (2019).

[8] Julien-Vergonjanne, A., Sahuguède, S., and Chevalier, L., [Optical Wireless Communications: An Emerging Technology], ch. Optical Wireless Body Area Networks for Healthcare Applications, 569-587, Springer (2016).

[9] Movassaghi, S., Abolhasan, M., Lipman, J., Smith, D., and Jamalipour, A., "Wireless body area networks: A survey," IEEE Communications Surveys Tutorials 16, 1658-1686 (Jan. 2014).

[10] Proakis, J. G. and Salehi, M., [Digital Communications], McGraw-Hill, New York, 5th ed. (2007).

[11] "Opticstudio." https://www.zemax.com/products/opticstudio. Accessed: 16-09-2019.

[12] Miramirkhani, F. and Uysal, M., "Channel modeling and characterization for visible light communications," IEEE Photonics Journal 7, 1-16 (Dec. 2015).

[13] Bettstetter, C., Hartenstein, H., and Pérez-Costa, X., "Stochastic properties of the random waypoint mobility model," Wireless Networks 10, 555-567 (Sep. 2004).

[14] Long, S., Khalighi, M. A., Wolf, M., Bourennane, S., and Ghassemlooy., Z., "Investigating channel frequency selectivity in indoor visible-light communication systems," IET Optoelectronics 10, 80-88(8) (June 2016).

[15] Epanechnikov, V., "Non-parametric estimation of a multivariate probability density," Theory of Probability and Its Applications 14, 153158 (Jan. 1969).

[16] Silverman, B., [Density Estimation for Statistics and Data Analysis], Chapman \& Hall/CRC, London (1986).

[17] Xu, F., Khalighi, M., and Bourennane, S., "Impact of different noise sources on the performance of PINand APD-based FSO receivers," in [International Conference on Telecommunications (ConTel)], 211-218 (June 2011). Graz, Austria. 